\title{
Effectiveness on mild stress and mixed urinary incontinence and impact on Quality of Life of a phytotherapic product containing astragalus, thyme, lavender, hop, equisetum, red clover, cypress and agrimonia at titrated concentrations. Results from a monocentric study
}

\author{
Oreste Risi ${ }^{1}$, Michele Manica ${ }^{1}$, Rocca Carmela Lisanti ${ }^{2}$, Antonio Manfredi ${ }^{1}$, Giuseppe Romeo Tecci ${ }^{1}$ \\ ${ }^{1}$ SSD Urodinamica e Andrologia, ASST Bergamo Ovest, Italy; \\ ${ }^{2}$ UO Urologia, Humanitas Cliniche Gavazzeni, Bergamo, Italy.
}

\begin{abstract}
Summary Objectives: To assess any beneficial effect on quality of life of a daily treatment with a phytotherapic product containing astragalus, thyme, lavender, hop, equisetum, red clover, cypress and agrimonia at titrated concentrations in a cohort of female patients complaining mild stress urinary incontinence (SUI) or mixed urinary incontinence (MUI).

Materials and methods: 42 non-consecutive female out-patients with mild SUI or mild MUI were assessed with a clinical evaluation, International Consultation on Incontinence

Questionnaire Short Form (ICIQ-SF) and Patients' Perception of Intensity of Urgency Scale (PPIUS) at baseline the start of the study and after two months of therapy with the phytotherapic product. At the end of the therapy the patients also compiled Patient Global Impression of Improvement (PGI-I). Results: After the completion of the study there was a trend towards better results in each item of ICIQ-SF, but without any statistical significance with an average score in ICIQ-SF-1 of $3.12 \pm 0.981$ versus $3.21 \pm 0.914(p=0.556)$, in ICIQ-SF-2 of $3.69 \pm 1.422$ versus $3.79 \pm 1.372(p=0.68)$ and in ICIQ-SF-3 of $5.95 \pm 1.618$ versus $6.14 \pm 1.670(p=0.462)$. The average reduction of PPIUS was of $0.09(1.26 \pm 1.481$ versus $1.357 \pm$ $1.509, p=0.705)$. There was a reduction of average consumption of pads/die from $1.69 \pm 0.636$ to $1.54 \pm 0.543(p=0.101)$. In relation to the PGI score, $23 / 42$ patients (54.7\%) reported no changes after the completion of the therapy, $13 / 42$ (30.9\%) reported a slight improvement, 5/42 (11.9\%) were much improved and 1/42 (2.3\%) was slightly worsened. Only 2/42 (4.7\%) patients discontinued the treatment before of the completion of the study. We did not observe any adverse effects during the period of the study.

Conclusions: The phytotherapic product seems to cause a slight improvement of the symptoms in a good rate of patients.

Moreover it has a low rate of withdrawal, due to the lack of adverse events.
\end{abstract}

KEY WORDS: Mixed urinary incontinence; Stress urinary incontinence; Phytoterapy; QoL.

Submitted 7 March 2017; Accepted 6 October 2017

\section{INTRODUCTION}

Stress Urinary Incontinence (SUI) is defined as the involuntary loss of urine on effort or physical exertion or on sneezing or coughing. Mixed urinary incontinence (MUI) is defined as the involuntary loss of urine associated with urgency or with effort or physical exertion or on sneezing or coughing (1). In most studies the reported prevalence in females of isolated SUI was of 10-39\%, accounting for about a half of all the cases of urinary incontinence. MUI is the second most common type, with reported prevalence of $7.5-25 \%$ in most studies (1). Therefore SUI and MUI represent an important and common problem involving many women with a high social impact (2).

Nowadays, according to European Association of Urology Guidelines, the first-line treatment for SUI is a supervised intensive pelvic floor muscle training (PMFT)

Biofeedback can be considered as an adjunct in these patients (3). Duloxetine $80 \mathrm{mg}$ was proposed as oral therapy for SUI but there is no evidence suggesting that it can be offered to women who are seeking a definitive cure for their incontinence. Furthermore Duloxetine had a high withdrawal rate primarily because of the high rate of adverse events (3-6). Therefore apart from the surgical treatment we have no other choice for SUI treatment after the PMFT and eventually the biofeedback.

In MUI, PMFT seems to be less effective than in pure SUI (7). Both Duloxetine and antimuscarinic drugs seem to be effective to treat SUI but it is known that most patients stop drugs because of low level of efficacy, onset of adverse events and high costs of therapy $(3,5,8)$.

IncontinenzaMEV ${ }^{\circledR} 1260 \mathrm{mg}$ is a phytotherapic product with antioxidant properties, proposed as natural remedy to induce physiological effects optimizing the functions of the urinary tract: microcirculation, relaxation, connective tissue tropism. The aim of this study is to assess any beneficial effect on the quality of life (QoL) of a daily treatment with IncontinenzaMEV ${ }^{\circledR}$ in a cohort of female patients complaining mild SUI or MUI.

\section{MATERIALS AND METHOdS}

From June 2015 to June 2016, 42 female non-consecutive out-patients with a median age of 58 years (range 
35-72, mean) referring to the Department of Urodynamics and Andrology of the ASST Bergamo Ovest and suffering from mild SUI or mild MUI were treated with IncontinenzaMEV ${ }^{\circledR}$ and prospectively evaluated.

Each patient was assessed before therapy (TO) with an accurate medical history, an urogynaecological examination, a comprehensive examination of urine including urine culture, a 3-day voiding diary, an evaluation of number of pads used per day, a 24-hour pad test, and a compilation of the International Consultation on Incontinence Questionnaire Short Form (ICIQ-SF).

Moreover each patient compiled the Patients' Perception of Intensity of Urgency Scale (PPIUS), a patient-reported instrument to measure the intensity of the urgency.

Inclusion criteria were: mild SUI or mild MUI defined as a range of urine loss for the 24-hour pad test from 4 to $20 \mathrm{~g}$, incontinence since at least 6 months, a positive stress-test.

Exclusion criteria were: positive urine culture, moderate and severe SUI (urine loss > $20 \mathrm{~g}$ for the 24-hour pad test), concomitant complicating factors as previous surgery for incontinence, previous pelvic radiotherapy, grade 3 or symptomatic prolapse, hematuria, pain or pelvic mass.

The 42 patients included in the study assumed IncontinenzaMEV $\mathrm{V}^{\circledR}$ one tablet/daily for two months.

IncontinenzaMEV ${ }^{\circledR} 1260 \mathrm{mg}$ is a phytotherapic product containing astragalus, thyme, lavender, hop, equisetum, red clover, cypress and agrimonia at titrated concentrations.

During the period of the study no other drugs for incontinence were administered.

At the end of the therapy (T1) all the patients were interviewed again and compiled again ICIQ-SF and PPIUS. Furthermore they filled the Patient Global Impression of Improvement (PGI-I), a l-item questionnaire to evaluate the subjective feeling of improvement.

The statistical analysis was performed using IBM SPSS Statictics 23. The comparison of the data relative to ICIQ-SF an PPIUS before and at the end of the therapy was performed using Wilcoxon Signed Ranks Test.

The comparison between the used pads was made with Student's T-Test. We considered statistically significant a $\mathrm{p}$ value $\leq 0.05$.

\section{Results}

Eight out of 42 (19\%) patients had a MUI with relevant urgency incontinence, evaluated by PPIUS; 34/42 (81\%) complained an isolated SUI with (17/34) or without (17/34) urgency symptoms.

After the completion of the study there was a trend towards better results in each item of ICIQ-SF, without any statistical significance with an average score in ICIQSF-1 of $3.12 \pm 0.981$ versus $3.21 \pm 0.914(\mathrm{p}=0.556)$, in ICIQ-SF-2 of $3.69 \pm 1.422$ versus $3.79 \pm 1.372(\mathrm{p}=$ $0.68)$ and in ICIQ-SF-3 of $5.95 \pm 1.618$ versus $6.14 \pm$ $1.670(\mathrm{p}=0.462)$.

Five out of 17 patients (29.4\%) who reported urgency symptoms before the beginning of the therapy, improved their PPIUS score, while 1/17 reported a worse value. The average reduction of PPIUS was of 0.09 (1.26 \pm 1.481 versus $1.357 \pm 1.509, \mathrm{p}=0.705)$.
Table 1.

Results of the ICIQ-SF, PPIUS, pads/die at $T_{0}$ and at $T_{1}$.

\begin{tabular}{|lcccc|}
\hline & T0 & T1 & $\Delta$ & P \\
\hline ICIQ-SF 1 & $3.21 \pm 0.914$ & $3.12 \pm 0.981$ & 0.09 & 0.556 \\
\hline ICIQ-SF 2 & $3.79 \pm 1.372$ & $3.69 \pm 1.422$ & 0.10 & 0.689 \\
\hline ICIQ-SF 3 & $6.14 \pm 1.670$ & $5.95 \pm 1.618$ & 0.19 & 0.462 \\
\hline PPIUS & $1.357 \pm 1.509$ & $1.26 \pm 1.481$ & 0.09 & 0.705 \\
\hline $\mathbf{n}$ pads & $1.69 \pm 0.636$ & $1.54 \pm 0.543$ & 0.15 & 0.101 \\
\hline
\end{tabular}

None of the 8 patients with a component of urge incontinence (defined as an initial PPIUS score $=4$ ) changed their initial value.

There was a reduction of average consumption of pads/die from $1.69 \pm 0.636$ to $1.54 \pm 0.543(\mathrm{p}=0.101)$.

These results are showed in Table 1 .

With regard to the PGI score, $23 / 42$ patients (54.7\%) reported no changes after the completion of the therapy, 13/42 (30.9\%) reported a slight improvement, 5/42 (11.9\%) were much improved and 1/42 (2.3\%) was slightly worsened. These results are described in Figure 1.

Only $2 / 42(4.7 \%)$ patient discontinued the treatment before of the completion of the study because of the lack of efficacy of treatment.

We did not observe any adverse effects during the period of the study.

\section{Figure 1.}

Frequency distribution of the answers to PGI-I questionnaire: "check the number that best describes how your post-treatment condition is now, compared with how it was before you had the treatment".

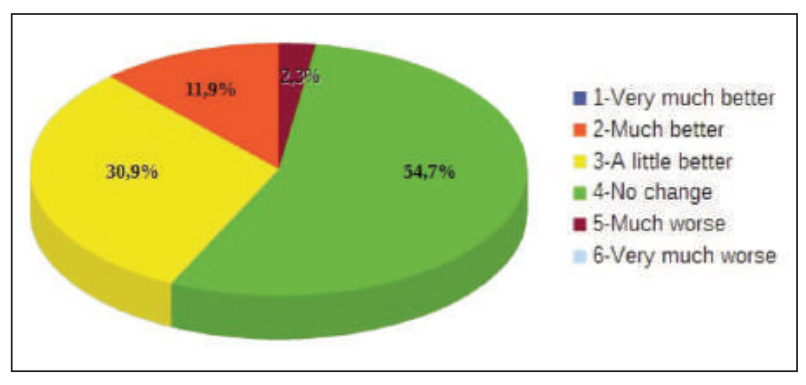

\section{Discussion}

Pharmacological treatment of stress and mixed urinary incontinence is still a hot topic because of the of low efficacy, the adverse events, and the high costs of the of the available drugs $(3,5,8)$.

Approximately $50 \%$ of patients in treatment with antimuscarinic drugs discontinue therapy at 3 months, mainly because of the relevant side effects (9).

In the last years Mirabegron was proposed for the use in overactive bladder with a grade B of recommendation (3). This drug seems to cause less dry mouth than anticholinergic medications, however the incidence of other side effects such as constipation, hypertension and tachycardia is comparable (10).

Duloxetine was proposed besides PMFT as oral therapy for SUI with controversial results and high withdrawal rate (5). 
In this study we evaluated the effects on MUI and SUI of a daily therapy with IncontinenzaMEV, a new phytotherapic product. The main limitations of our study are monocentric design, low number of patients and lack of a placebo control group. Given these limitations we observed a slight improvement in $30.9 \%$ of patients treated with the study product and a more consistent improvement in 5 patients of the series (11.9\% of subjects.).

These results are inferior respect to a recent study on Mirabegron which prospectively evaluated PGI-I in 317 women after 6 weeks of drug therapy: 11\% described themselves as "very much better" and a further 23\% as "much better". Anyway the target of this study was different because only women with overactive bladder (OAB) were selected. Moreover there was a higher rate of withdrawal (14.6\%) (11).

The same considerations are possible for another study which evaluated the PGI scores in a series of 50 women patients treated with duloxetine for a pure SUI: $65 \%$ showed improvement after a 12 weeks-treatment but the group was more homogeneous than ours. Moreover also in this series there was a higher number of dropouts and adverse events (12). In our study all the questionnaires administrated showed a trend towards better scores after the two months of therapy but we had not a statistical significance. The more evident improvement was relative to the QoL (ICIQ-SF-3). Conversely an important result is the low rate of withdrawal during all the period of the study, probably due to the lack of adverse effects of this remedy for incontinence.

\section{Conclusions}

To our knowledge this is the first study on IncontinenzaMEV. With the limits of a small sample of patients and of the lack of a control group this product seems to cause a slight improvement of the symptoms in a good rate of patients. Moreover unlike other drugs it has a very low rate of withdrawal. Further studies with a control group are warranted to offer a recommendation and to better assess the target of this product.

\section{REFERENCES}

1. Abrams P, Cardozo L, Khoury S, Wein A. Incontinence. 5th edition, ICUD-EAU 2013.

2. Cervigni M, Gambacciani M. Female urinary stress incontinence. Climacteric. 2015; 18 Suppl 1:30-6.

3. Burkhard FC, Lucas MG, Berghmans JL, et al. EAU Guidelines on Urinary Incontinence in Adults. European Association of Urology 2016.

4. Shamliyan TA, Kane RL, Wyman J, Wilt TJ. Systematic review: randomized, controlled trials of nonsurgical treatments for urinary incontinence in women. Ann Intern Med. 2008; 148:459-73.

5. Mariappan P, Alhasso A, Ballantyne Z, et al. Duloxetine, a serotonin and noradrenaline reuptake inhibitor (SNRI) for the treatment of stress urinary incontinence: a systematic review. Eur Urol. 2007; 51:67-74.

6. Ghoniem GM, Van Leeuwen JS, Elser DM, et al. A randomized controlled trial of duloxetine alone, pelvic floor muscle training alone, combined treatment and no active treatment in women with stress urinary incontinence. J Urol. 2005; 173:1647-53.

7. Lagro-Janssen T, van Weel C. Long-term effect of treatment of female incontinence in general practice. Br J Gen Pract. 1998; 48:1735-8.

8. Sears CL, Lewis C, Noel K, et al. Overactive bladder medication adherence when medication is free to patients. J Urol. 2010; 183:1077-1081.

9. Wagg A, Compion G, Fahey A, Siddiqui E. Persistence with prescribed antimuscarinic therapy for overactive bladder: a UK experience. BJU Int. 2012; 110:1767-74.

10. Warren K, Burden H, Abrams P. Mirabegron in overactive bladder patients: efficacy review and update on drug safety Ther $A d v$ Drug Saf. 2016; 7:204-6.

11. Balachandran A, Duckett J. The efficacy and tolerability of mirabegron in a non-trial clinical setting. Eur J Obstet Gynecol Reprod Biol. 2016; 200:63-7.

12. Deepak P, Kumar TN, Sen TK. Evaluation of efficacy of duloxetine in stress urinary incontinence in women. Indian J Pharmacol. 2011; 43:176-9.

\author{
Correspondence \\ Oreste Risi, MD (Corresponding Author) \\ oreste_risi@asst-bgovest.it \\ Michele Manica, MD, FEBU \\ manicaxmichele@gmail.com \\ Antonio Manfredi, MD \\ antonio_manfredi@asst-bgovest.it \\ Giuseppe Romeo Tecci, MD \\ rom.doc@libero.it \\ SSD Urodinamica e Andrologia, ASST Bergamo Ovest \\ Piazzale Ospedale 1, 24047 Treviglio (BG), Italy \\ Rocca Carmela Lisanti, MD \\ rocca.lisanti@gavazzeni.it \\ UO Urologia, Humanitas Cliniche Gavazzeni \\ Via Mauro Gavazzeni 21, 24125 Bergamo (BG), Italy
}

